\title{
Psychological Aspects of Students With Learning Disabilities in E-Environments: A Mini Review and Future Research Directions
}

\author{
Stefania Cataudella*, Stefano Carta, Maria Lidia Mascia, Carmelo Masala, \\ Donatella Rita Petretto and Maria Pietronilla Penna
}

Department of Pedagogy, Psychology, Philosophy, University of Cagliari, Cagliari, Italy

\section{OPEN ACCESS}

Edited by:

Gabriella Martino,

University of Messina, Italy

Reviewed by:

Luana Sorrenti,

University of Messina, Italy

Leslie D. Frazier,

Florida International University,

United States

*Correspondence:

Stefania Cataudella

scataude/@unica.it

Specialty section:

This article was submitted to

Psychopathology,

a section of the journal

Frontiers in Psychology

Received: 29 September 2020 Accepted: 01 December 2020 Published: 07 January 2021

Citation:

Cataudella S, Carta S,

Mascia ML, Masala C, Petretto DR and Penna MP (2021) Psychological

Aspects of Students With Learning Disabilities in E-Environments: A Mini

Review and Future Research

Directions.

Front. Psychol. 11:611818. doi: 10.3389/fpsyg.2020.611818
What are the main learning difficulties or advantages encountered by students with learning disabilities (LDs) within e-environments? As a result of the Covid-19 emergency, e-learning is being increasingly used to support students' learning processes. A number of countries closed their schools altogether, so face-to-face lessons were and have been replaced by distance lessons. A search of current literature via Scopus, Eric and Google Scholar electronic databases was conducted according to Prisma Guidelines. Other sources of literature were also considered, starting from the references in the full text of the articles consulted. We used the following search keywords: "LDs" combined with the "AND/OR" Boolean operator and "e-learning platforms," "well-being," "psychological factors," "emotional distress," and "self-regulation." One body of literature highlights the lack of inclusive accessibility standards and a lack of attention to specific tools for addressing LDs, which causes students to develop high levels of stress/anxiety and emotional distress, in addition to low levels of well-being, self-esteem and self-efficacy. Another area of literature looks at how students can develop high levels of self-regulation and emotional awareness, as well as high levels of inclusion. Results are discussed in terms of the promotion of e-learning that focuses on the psychological well-being of students and teachers use of technological tools.

Keywords: e-learning, psychological well-being, emotional distress, self-regulation, learning disabilities

\section{INTRODUCTION}

The forced interruption of face-to-face teaching due to the worldwide outbreak of Covid-19, has significantly reactivated the debate on the concrete effectiveness and functionality of e-learning courses. Specifically, our goal was to better understand the psychological effects and efficacy of the current massive use of the e-environments on students with learning disabilities (LDs) (Viner et al., 2020). Literature shows a variety of ways to define e-learning. For example, Cidral et al. (2018) define e-learning as a web-based learning system for the dissemination of information, communication, and knowledge for education and training. Until 2002, Eletti had affirmed that e-learning is a new type of training, a new teaching system that allows you to follow and above all personalize learning. The services and tools used allow for continuous contact with the "student". In addition, a platform and an interface built ad hoc, adapting the contents, allows to model the 
teaching on the user's needs (Eletti, 2002). Thus, in light of the massive use of e-environments, there is a definite need to question how effective these tools are for students with LDs. According to international diagnostic criteria, LDs are an overarching group of neurodevelopmental disorders comprising different learning disorders that affect primary and/or secondary academic abilities and a child's overall capabilities (American Psychiatric Association, 2013; Schulte-Korne, 2014). Children with specific LDs are a rather heterogeneous group, both with regard to specific academic abilities such as listening, thinking, reading, speaking, writing, calculating, and spelling (Sorrenti et al., 2019), as well as to their neuropsychological and functional profiles. For example, they may have impairments affecting different cognitive and neuropsychological abilities (working memory), long-term memory (implicit and explicit memory), attention (selective and sustained), and linguistic, praxis, visuospatial, problem solving, and/or executive abilities (Petretto and Masala, 2017; Visser et al., 2020), etc. Moreover, there is general agreement on the association between LDs and other neurodevelopmental disorders (ADHD and specific language disorders); LDs typically occur in individuals of normal intelligence (Sorrenti et al., 2019). A body of studies indicates a relationship between children's LD and poor social relations in school (Walker and Nabuzoka, 2007), this aspect is confirmed also in the University context (Filippello et al., 2019). Literature shows a relationship between LDs and internalizing (depressive and anxiety disorders) and externalizing disorders (conduct disorders) (Frith, 2013; Bonifacci et al., 2016; Panicker and Chelliah, 2016; Visser et al., 2020). If LDs are not adequately treated, they can evolve over time, potentially resulting in forms of psycho-social maladjustment (Sorrenti et al., 2019). Regarding the use of e-learning, only a small number of studies have addressed these psychological factors and consequences, and there are few studies which have directly examined the quality of life of students with LDs, or the quality of interpersonal relationships (parents, teachers, and peers). In this mini-review and according to previous research in the field, we analyze these aspects and focus our attention to the following questions:

(1) What are the effects of the use of e-learning on psychological well-being?

(2) What are the effects of accessibility standards in promoting inclusion and in reducing stress, anxiety and emotional distress among students with LDs?

\section{METHODOLOGY}

A search of current literature using Scopus, Eric and Google Scholar electronic databases was conducted according to Prisma Guidelines (Moher et al., 2015). Other sources of literature were also considered, starting from the references in the full texts of the articles examined. We used the following search keywords: "LDs" combined with the "AND/OR" Boolean operator and "e-learning platforms," "well-being," "psychological factors," "emotional distress", and "self-regulation". Applying a systematic procedure, literature was then selected and results were charted and analyzed. The following inclusion criteria were established: papers on the use of e-learning with LD; on the relationship between e-learning platforms and related psychological aspects (self-esteem, emotional distress, and selfregulation); written in English and published from 2015 to 2020. The following exclusion criteria were applied: systematic reviews; papers on the use of e-learning without LD. On the basis of the research questions and the literature considered, we chose a minireview. For this reason the data will be presented as a narrative review.

\section{RESULTS AND DISCUSSION}

In the first part of the search, two independent assessors found 53 articles. Applying our inclusion and exclusion criteria, after reading the abstract, 27 articles were considered. After reading the full texts, 4 further articles were excluded, thus a final group of 23 articles were considered (Table 1). As expected, in literature, regarding the definition of "e-learning", we found different systems and tools (platforms, devices, web materials/sites, Learning Content Management Systems, ICT, etc.). According to Bjekic et al. (2014) we categorized the different definitions in two groups. The first group refers to the use of Assistive Technology (AT) (hardware or software, used to increase, improve or maintain capabilities of persons with LDs aimed to support and/or increase learning). The second group of e-learning refers to a system of procedures, processes and instructional materials that supports learning. Moreover, we considered a difference between e-platforms and ICT tools (Salehi et al., 2015; Table 2).

The papers showed a certain amount of heterogeneity in their definition of LDs. Some authors proposed a specific definition (Chen et al., 2015; Richardson, 2015; Shonfeld and Ronen, 2015; Straub and Vasquez, 2015; Benmarrakchi et al., 2017; Sharabi et al., 2016; Adam and Tatnall, 2017; Vasalou et al., 2017; Lambert and Dryer, 2018; Lipka et al., 2019; Ziadat, 2019), while others proposed a general reference to Special Educational Needs or used the World Health Organization definition of Disability (World Health Organization, 2001; Berizzi et al., 2017; Naumova et al., 2017; García-González et al., 2020). Some papers reported the definition of LD based on international diagnostic criteria, others described specific national law/s or references (Sharabi et al., 2016). Moreover, with regard to sample recruitment, some authors chose samples consisting of different groups of students with other kinds of disabilities and then specified the number of students with LDs (Richardson, 2015, 2016; Shonfeld and Ronen, 2015; Terras et al., 2015; Benmarrakchi et al., 2017; Sharabi et al., 2016; Alamri and Tyler-Wood, 2017; Berizzi et al., 2017; Kent et al., 2018; Lipka et al., 2019; Ouherrou et al., 2019; GarcíaGonzález et al., 2020); while in other papers, the sample is made up only of students with LDs (Chen et al., 2015; Straub and Vasquez, 2015; Vasalou et al., 2017; Lambert and Dryer, 2018). Regarding the level of schooling, about $1 / 2$ of the studies focused on University environments (Richardson, 2015, 2016; Terras et al., 2015; Alamri and Tyler-Wood, 2017; Naumova et al., 2017; Kent et al., 2018; García-González et al., 2020) and the 
TABLE 1 | Characteristics of papers which met the inclusion criteria

\begin{tabular}{|c|c|c|c|c|c|c|c|c|c|c|c|}
\hline $\begin{array}{l}\text { Author(s), } \\
\text { Year }\end{array}$ & $\begin{array}{l}\text { Topic of } \\
\text { paper }\end{array}$ & $\begin{array}{l}\text { Country/ } \\
\text { Countries }\end{array}$ & $\begin{array}{l}\text { Point } \\
\text { of view }\end{array}$ & Accessibility & Methodology & $\begin{array}{l}\text { Definition e-learning } \\
\text { platforms, } \\
\text { instruments and/or } \\
\text { devices used }\end{array}$ & $\begin{array}{l}\text { Total no. of } \\
\text { subjects } \\
\text { (Total no. of } \\
\text { subjects with } \\
\text { LD) }\end{array}$ & $\begin{array}{l}\text { Type of } \\
\text { school/ } \\
\text { university }\end{array}$ & Kind of LD & Age range & $\begin{array}{l}\text { Assessed } \\
\text { Variables }\end{array}$ \\
\hline $\begin{array}{l}\text { 1. Adam } \\
\text { and Tatnall, } \\
2017\end{array}$ & $\begin{array}{l}\text { This study investigates } \\
\text { whether, and if so how, } \\
\text { ICT could be used to } \\
\text { support school } \\
\text { communities involving } \\
\text { students with learning } \\
\text { difficulties, and whether } \\
\text { it could help these } \\
\text { students with their } \\
\text { learning in two special } \\
\text { School settings }\end{array}$ & Australia & $\mathrm{T} / \mathrm{P}$ & No & $\begin{array}{l}\text {-Case studies } \\
\text {-Interviews with the } \\
\text { School Principal, } \\
\text { Teachers and Parents }\end{array}$ & $\begin{array}{l}\text { ICT in teaching -ICT } \\
\text { was used } \\
\text { predominantly to } \\
\text { reinforce language and } \\
\text { numeracy skills } \\
\text {-Classrooms are } \\
\text { equipped with an } \\
\text { electronic whiteboard } \\
\text { and each student has } \\
\text { access to a notebook } \\
\text { computer and iPad. ICT } \\
\text { offered opportunities for } \\
\text { students to use } \\
\text { technology that would } \\
\text { improve their literacy } \\
\text { output, access and } \\
\text { exposure to technology } \\
\text { as well as increasing } \\
\text { engagement and } \\
\text { provides evidence that } \\
\text { scaffolding with a direct } \\
\text { teaching approach } \\
\text { enhances the learning } \\
\text { outcomes of LD } \\
\text { students. }\end{array}$ & $\begin{array}{l}-180 \text { students } \\
\text { in the first } \\
\text { school } \\
\text {-About } \\
400 \text { students in } \\
\text { the second } \\
\text { school } \\
\text { (n.s.) }\end{array}$ & $S$ & n.s. & n.s. & $\begin{array}{l}\text {-Attainment in skills } \\
\text { and academic } \\
\text { knowledge -use of } \\
\text { ICT enhances LD } \\
\text { students' } \\
\text { independence and } \\
\text { equips them with } \\
\text { adequate skills } \\
\text { which should allow } \\
\text { them to continue } \\
\text { with further study } \\
\text { through various } \\
\text { pathways and to } \\
\text { move into a normal } \\
\text { work environment }\end{array}$ \\
\hline $\begin{array}{l}\text { 2. Alamri } \\
\text { and } \\
\text { Tyler-Wood, } \\
2017\end{array}$ & $\begin{array}{l}\text { This study investigates } \\
\text { which factors } \\
\text { associated with } \\
\text { learners with disabilities } \\
\text { impact student } \\
\text { outcomes in an online } \\
\text { learning -Environment } \\
\text { Successes and } \\
\text { struggles in an online } \\
\text { setting -Nature of } \\
\text { interaction between } \\
\text { students with } \\
\text { disabilities and } \\
\text { instructors }\end{array}$ & $\begin{array}{l}\text { USA- } \\
\text { midwest }\end{array}$ & S & No & $\begin{array}{l}\text { Electronic survey of } 20 \\
\text { questions }\end{array}$ & $\begin{array}{l}\text { For online courses, the } \\
\text { interaction can take } \\
\text { place through the use } \\
\text { of both synchronous } \\
\text { tools } \\
\text { (videoconferencing, } \\
\text { audio stream, online } \\
\text { chat sessions) and } \\
\text { asynchronous tools } \\
\text { (e-mail, discussion } \\
\text { boards). }\end{array}$ & $\begin{array}{l}40 \text { (4LD, } 10 \\
\text { ADHD) }\end{array}$ & $u$ & LD and ADHD & $18-58$ & $\begin{array}{l}\text {-Social presence, } \\
\text {-interpersonal } \\
\text { relationships } \\
\text {-Achievement } \\
\text {-Satisfaction } \\
\text {-different types of } \\
\text { interactions in } \\
\text { online learning: } \\
\text { learner-interface } \\
\text { interaction, } \\
\text { learner-content } \\
\text { interaction, and } \\
\text { learner-learner } \\
\text { interaction. }\end{array}$ \\
\hline
\end{tabular}


TABLE 1 | Continued

\begin{tabular}{|c|c|c|c|c|c|c|c|c|c|c|c|}
\hline $\begin{array}{l}\text { Author(s), } \\
\text { Year }\end{array}$ & $\begin{array}{l}\text { Topic of } \\
\text { paper }\end{array}$ & $\begin{array}{l}\text { Country/ } \\
\text { Countries }\end{array}$ & $\begin{array}{l}\text { Point } \\
\text { of view }\end{array}$ & Accessibility & Methodology & $\begin{array}{l}\text { Definition e-learning } \\
\text { platforms, } \\
\text { instruments and/or } \\
\text { devices used }\end{array}$ & $\begin{array}{l}\text { Total no. of } \\
\text { subjects } \\
\text { (Total no. of } \\
\text { subjects with } \\
\text { LD) }\end{array}$ & $\begin{array}{l}\text { Type of } \\
\text { school/ } \\
\text { university }\end{array}$ & Kind of LD & Age range & $\begin{array}{l}\text { Assessed } \\
\text { Variables }\end{array}$ \\
\hline $\begin{array}{l}\text { 3. Baharuddin and } \\
\text { Dalle, } 2019\end{array}$ & $\begin{array}{l}\text { The study uses a } \\
\text { four-phase iterative } \\
\text { process to develop and } \\
\text { analyze a prototype } \\
\text { eLearning system: } \\
\text { understanding the } \\
\text { problem, designing the } \\
\text { system, developing the } \\
\text { system, and gathering } \\
\text { user feedback }\end{array}$ & Indonesia & $\mathrm{T} / \mathrm{S}$ & No & $\begin{array}{l}\text { Interviewed observation } \\
\text { during the use of ICT }\end{array}$ & $\begin{array}{l}\text { Computer connected } \\
\text { to the Internet, virtual } \\
\text { classrooms }\end{array}$ & $\begin{array}{l}17 \text { teachers } \\
\text { (n.s.) }\end{array}$ & $S$ & n.s. & n.s. & $\begin{array}{l}\text { Communication } \\
\text { Attendance } \\
\text { Reasonable } \\
\text { accommodations } \\
\text { Knowledge and } \\
\text { competencies of } \\
\text { teachers }\end{array}$ \\
\hline $\begin{array}{l}\text { 4. Benmarrakchi } \\
\text { et al., } 2017\end{array}$ & $\begin{array}{l}\text { This study investigates } \\
\text { the potential benefits } \\
\text { offered by the use of } \\
\text { Information and } \\
\text { Communication } \\
\text { Technology (ICT) to } \\
\text { support dyslexic } \\
\text { students by considering } \\
\text { their preferred learning } \\
\text { styles. Based on the } \\
\text { results of the analysis of } \\
\text { learning styles } \\
\text { differences, the authors } \\
\text { introduced an adaptive } \\
\text { mobile learning to } \\
\text { support and promote } \\
\text { learning for dyslexic } \\
\text { students. }\end{array}$ & Morocco & $S$ & No & Questionnaires & $\begin{array}{l}\text { ICT (digital technologies } \\
\text { Multimedia } \\
\text { applications) adaptive } \\
\text { mobile learning }\end{array}$ & $28(8)$ & PS & dyslexia & $8-10$ & $\begin{array}{l}\text { Learning styles } \\
\text { (description of the } \\
\text { attitudes and } \\
\text { behaviors, which } \\
\text { determine an } \\
\text { individual's } \\
\text { preferred way of } \\
\text { learning VAK } \\
\text { learning style model } \\
\text { is based on three } \\
\text { main sensory } \\
\text { receivers: visual (N), } \\
\text { auditory (A), and } \\
\text { kinesthetic (K). } \\
\text { - 'cycle of learning', } \\
\text { four-stage cycle of } \\
\text { learning: abstract } \\
\text { conceptualization } \\
\text { (AC), concrete } \\
\text { experience (CE), } \\
\text { active } \\
\text { experimentation } \\
\text { (AE), and reflective } \\
\text { observation (RO). }\end{array}$ \\
\hline
\end{tabular}

(Continued) 
TABLE 1 | Continued

\begin{tabular}{|c|c|c|c|c|c|c|c|c|c|c|c|}
\hline $\begin{array}{l}\text { Author(s), } \\
\text { Year }\end{array}$ & $\begin{array}{l}\text { Topic of } \\
\text { paper }\end{array}$ & $\begin{array}{l}\text { Country/ } \\
\text { Countries }\end{array}$ & $\begin{array}{l}\text { Point } \\
\text { of view }\end{array}$ & Accessibility & Methodology & $\begin{array}{l}\text { Definition e-learning } \\
\text { platforms, } \\
\text { instruments and/or } \\
\text { devices used }\end{array}$ & $\begin{array}{l}\text { Total no. of } \\
\text { subjects } \\
\text { (Total no. of } \\
\text { subjects with } \\
\text { LD) }\end{array}$ & $\begin{array}{l}\text { Type of } \\
\text { school/ } \\
\text { university }\end{array}$ & Kind of LD & Age range & $\begin{array}{l}\text { Assessed } \\
\text { Variables }\end{array}$ \\
\hline $\begin{array}{l}\text { 5. Berizzi } \\
\text { et al., } 2017\end{array}$ & $\begin{array}{l}\text { This study investigates } \\
\text { the effect of an } \\
\text { attributive- } \\
\text { metacognitive training } \\
\text { on attributional style of } \\
\text { students with Special } \\
\text { Educational Needs } \\
\text { (SEN), proposed by a } \\
\text { elearning platform }\end{array}$ & Italy & S & Yes & Questionnaires & $\begin{array}{l}\text { ICT (e-mail, Skype } \\
\text { network conversations, } \\
\text { videoconferencing, } \\
\text { e-learning platforms, } \\
\text { such as Moodle, } \\
\text { Edmodo, and others) }\end{array}$ & $\begin{array}{l}30 \text { students } \\
\text { with special } \\
\text { educational } \\
\text { needs (30) }\end{array}$ & SS & $\begin{array}{l}24 \text { dyslexia } 6 \\
\text { ADHD }\end{array}$ & $11-15$ & $\begin{array}{l}\text { Metacognition Self } \\
\text { esteem Self efficacy } \\
\text { Locus of control }\end{array}$ \\
\hline $\begin{array}{l}\text { 6. Chen } \\
\text { et al., } 2015\end{array}$ & $\begin{array}{l}\text { This study aims to } \\
\text { explore the learning } \\
\text { experience of learners } \\
\text { with dyslexia when } \\
\text { reading passages using } \\
\text { different online reading } \\
\text { affordances to derive } \\
\text { some guidelines for } \\
\text { dyslexia-friendly online } \\
\text { text. }\end{array}$ & Malaysia & S & $\begin{array}{l}\text { Yes Web site } \\
\text { accessibility } \\
\text { guidelines } \\
\text { and role of } \\
\text { engagement }\end{array}$ & $\begin{array}{l}\text { Qualitative } \\
\text { multiple-case study }\end{array}$ & $\begin{array}{l}\text { Web sites The use of } \\
\text { online learning is } \\
\text { appropriate for learners } \\
\text { with dyslexia as this } \\
\text { delivery mode allows } \\
\text { self-paced learning and } \\
\text { affords multimodal } \\
\text { technologies that have } \\
\text { the potential to settle } \\
\text { dominant deficit } \\
\text { models of dyslexia }\end{array}$ & $12(12)$ & SS & Dyslexia & $14-18$ & $\begin{array}{l}\text { Online reading } \\
\text { affordances } \\
\text { Perceived learning } \\
\text { behavioral } \\
\text { engagement (BE), } \\
\text { cognitive } \\
\text { engagement (CE) } \\
\text { as well as affective } \\
\text { engagement (AE) } \\
\text { web accessibility } \\
\text { guidelines for users } \\
\text { with dyslexia }\end{array}$ \\
\hline $\begin{array}{l}\text { 7. García- } \\
\text { González } \\
\text { et al., } 2020\end{array}$ & $\begin{array}{l}\text { This study investigates } \\
\text { how students } \\
\text { perceived access to } \\
\text { higher education } \\
\text {-Role of barriers }\end{array}$ & Spanish & S & Yes & $\begin{array}{l}\text { Focus group and } \\
\text { interviews Discourse } \\
\text { analysis }\end{array}$ & $\begin{array}{l}\text { Personal Learning } \\
\text { Environments PLE } \\
\text { WEB }\end{array}$ & $16(3)$ & U & Dyslexia & $20-29$ & $\begin{array}{l}\text { Web or computer } \\
\text { barriers, Learning } \\
\text { barriers, burocratic } \\
\text { barriers, } \\
\text { architectural } \\
\text { barriers, social } \\
\text { barriers }\end{array}$ \\
\hline
\end{tabular}


TABLE 1 | Continued

\begin{tabular}{|c|c|c|c|c|c|c|c|c|c|c|c|}
\hline $\begin{array}{l}\text { Author(s), } \\
\text { Year }\end{array}$ & $\begin{array}{l}\text { Topic of } \\
\text { paper }\end{array}$ & $\begin{array}{l}\text { Country/ } \\
\text { Countries }\end{array}$ & $\begin{array}{l}\text { Point } \\
\text { of view }\end{array}$ & Accessibility & Methodology & $\begin{array}{l}\text { Definition e-learning } \\
\text { platforms, } \\
\text { instruments and/or } \\
\text { devices used }\end{array}$ & $\begin{array}{l}\text { Total no. of } \\
\text { subjects } \\
\text { (Total no. of } \\
\text { subjects with } \\
\text { LD) }\end{array}$ & $\begin{array}{l}\text { Type of } \\
\text { school/ } \\
\text { university }\end{array}$ & Kind of LD & Age range & $\begin{array}{l}\text { Assessed } \\
\text { Variables }\end{array}$ \\
\hline $\begin{array}{l}\text { 8. Kent } \\
\text { et al., } 2018\end{array}$ & $\begin{array}{l}\text { This study investigates } \\
\text { both the attitudes of } \\
\text { students with disability } \\
\text { towards disclosure of } \\
\text { their disabilities and } \\
\text { their experiences } \\
\text { ofstudying online and } \\
\text { accessibility of online } \\
\text { learning materials }\end{array}$ & Australia & S & $\begin{array}{l}\text { Yes } \\
\text { Accessibility } \\
\text { on web } \\
\text { materials } \\
\text { Need for a } \\
\text { more interest } \\
\text { toward } \\
\text { Universal } \\
\text { Design }\end{array}$ & Online survey & $\begin{array}{l}\text { E-learning in open } \\
\text { university }\end{array}$ & $\begin{array}{l}2000 \text { students } \\
\text { with disability } \\
\text { (16,3\% with LD) }\end{array}$ & $U$ & & Mean age 36 & $\begin{array}{l}\text { Accomodations } \\
\text { and disclosure of } \\
\text { disabilities and the } \\
\text { difficulties for } \\
\text { students to } \\
\text { disclosure their } \\
\text { disabilities, even if } \\
\text { this makes difficult } \\
\text { to personalized } \\
\text { accomodations. }\end{array}$ \\
\hline $\begin{array}{l}\text { 9. Lambert } \\
\text { and Dryer, } \\
2018\end{array}$ & $\begin{array}{l}\text { This study investigates } \\
\text { the effects of learning } \\
\text { challenges on online } \\
\text { learning environments } \\
\text { on the quality of life of } \\
\text { students with learning } \\
\text { disabilities }\end{array}$ & Australia & S & Yes & $\begin{array}{l}\text { Semi-structured } \\
\text { interview }\end{array}$ & $\begin{array}{l}\text { Online learning } \\
\text { environments }\end{array}$ & $8(8)$ & $U$ & Dyslexia & $21-43$ & $\begin{array}{l}\text { Stress, quality of } \\
\text { life, anxiety, self } \\
\text { esteem }\end{array}$ \\
\hline $\begin{array}{l}\text { 10. Lipka } \\
\text { et al., } 2019\end{array}$ & $\begin{array}{l}\text { This study investigated } \\
\text { how students and } \\
\text { instructors perceived } \\
\text { the instruction in } \\
\text { adapted courses in } \\
\text { post secondary school } \\
\text { students }\end{array}$ & Israel & $\mathrm{S} / \mathrm{T}$ & No & $\begin{array}{l}\text { Semi- } \\
\text { structuredinterviews }\end{array}$ & Adapted courses & $1000(5)$ & $U$ & LD and ADHD & 20 & $\begin{array}{l}\text { Perception of } \\
\text { teaching Locus of } \\
\text { control emotional } \\
\text { support }\end{array}$ \\
\hline $\begin{array}{l}11 . \\
\text { Naumova } \\
\text { et al., } 2017\end{array}$ & $\begin{array}{l}\text { This study investigates } \\
\text { an integrated } \\
\text { educational methods of } \\
\text { training matching the } \\
\text { features of disabled } \\
\text { students. The } \\
\text { technology includes } \\
\text { both traditional and } \\
\text { innovative methods of } \\
\text { training }\end{array}$ & Russia & S & Yes & n.s. & $\begin{array}{l}\text { Adapted courses with } \\
\text { online web content. } \\
\text { Information } \\
\text { technologies Web } \\
\text { content has to be } \\
\text { available for a wide } \\
\text { range of users with } \\
\text { health limitations. }\end{array}$ & 6 (n.s.) & $U$ & n.s. & n.s. & $\begin{array}{l}\text { Educational } \\
\text { Motivation } \\
\text { Interpersonal } \\
\text { Relationship }\end{array}$ \\
\hline $\begin{array}{l}\text { 12. Nieto- } \\
\text { Márquez } \\
\text { et al., } 2020\end{array}$ & $\begin{array}{l}\text { This study investigates } \\
\text { effects of digital } \\
\text { teaching on } \\
\text { metacognitive skills }\end{array}$ & Spain & S & No & Questionnaire & $\begin{array}{l}\text { Digital teaching } \\
\text { platform called Smile } \\
\text { and Learn, }\end{array}$ & 130 (n.s.) & PS & n.s. & 8 & Metacognitive skills \\
\hline
\end{tabular}


TABLE 1 | Continued

\begin{tabular}{|c|c|c|c|c|c|c|c|c|c|c|}
\hline $\begin{array}{l}\text { Author(s), } \\
\text { Year }\end{array}$ & $\begin{array}{l}\text { Topic of } \\
\text { paper }\end{array}$ & $\begin{array}{l}\text { Country/ } \\
\text { Countries }\end{array}$ & $\begin{array}{l}\text { Point } \\
\text { of view }\end{array}$ & Accessibility Methodology & $\begin{array}{l}\text { Definition e-learning } \\
\text { platforms, } \\
\text { instruments and/or } \\
\text { devices used }\end{array}$ & $\begin{array}{l}\text { Total no. of } \\
\text { subjects } \\
\text { (Total no. of } \\
\text { subjects with } \\
\text { LD) }\end{array}$ & $\begin{array}{l}\text { Type of } \\
\text { school/ } \\
\text { university }\end{array}$ & Kind of LD & Age range & $\begin{array}{l}\text { Assessed } \\
\text { Variables }\end{array}$ \\
\hline $\begin{array}{l}13 . \\
\text { Ouherrou } \\
\text { et al., } 2019\end{array}$ & $\begin{array}{l}\text { This study explored the } \\
\text { benefits of ICT use to } \\
\text { identify the ways in } \\
\text { which emotions are } \\
\text { involved during the } \\
\text { learning process in } \\
\text { Virtual Learning } \\
\text { Environments (VLE) }\end{array}$ & Morocco & S & Yes & $\begin{array}{l}\text { ICT Virtual learning } \\
\text { environment Artificial } \\
\text { intelligence Educational } \\
\text { games }\end{array}$ & $42(14)$ & PS & LD & $7-11$ & $\begin{array}{l}\text { Affective state } \\
\text { Emotions } \\
\text { Facial expression } \\
\text { recognition }\end{array}$ \\
\hline $\begin{array}{l}\text { 14. Rice } \\
\text { and Carter, } \\
2016\end{array}$ & $\begin{array}{l}\text { This study investigates } \\
\text { how practicing } \\
\text { teachers provided } \\
\text { self-regulation } \\
\text { strategies to students } \\
\text { with disabilities in a fully } \\
\text { online learning } \\
\text { environment. In this } \\
\text { context, the teachers } \\
\text { intended to offer } \\
\text { self-regulation } \\
\text { strategies to students, } \\
\text { but they were largely } \\
\text { unable to do so. }\end{array}$ & Kansas & $\mathrm{T}$ & Yes & E-learning environment & Teachers (n.s.) & $S$ & n.s. & n.s. & $\begin{array}{l}\text { Emotional } \\
\text { demands } \\
\text { Selfregulation } \\
\text { In students with } \\
\text { disability }\end{array}$ \\
\hline $\begin{array}{l}15 . \\
\text { Richardson, } \\
2015\end{array}$ & $\begin{array}{l}\text { This investigation } \\
\text { studied attainment in } \\
\text { students with dyslexia } \\
\text { or other specific } \\
\text { learning difficulties who } \\
\text { were taking modules by } \\
\text { distance learning with } \\
\text { the Open University in } \\
\text { 2012. Students with } \\
\text { dyslexia or other } \\
\text { specific learning } \\
\text { difficulties who had no } \\
\text { additional disabilities } \\
\text { were just as likely as } \\
\text { nondisabled students } \\
\text { to complete their } \\
\text { modules, but they were } \\
\text { less likely to pass the } \\
\text { modules that they had } \\
\text { completed and less } \\
\text { likely to obtain good } \\
\text { grades on the modules } \\
\text { that they had passed. }\end{array}$ & UK & S & Yes & $\begin{array}{l}\text { Distance learning in } \\
\text { open university } \\
\text { computer-based } \\
\text { support, particularly } \\
\text { CD-ROMs, dedicated } \\
\text { websites and } \\
\text { computer-mediated } \\
\text { conferencing }\end{array}$ & 4961 (n.s.) & $U$ & Dyslexia & $21-60$ & attainment \\
\hline
\end{tabular}


TABLE 1 | Continued

\begin{tabular}{|c|c|c|c|c|c|c|c|c|c|c|c|}
\hline $\begin{array}{l}\text { Author(s), } \\
\text { Year }\end{array}$ & $\begin{array}{l}\text { Topic of } \\
\text { paper }\end{array}$ & $\begin{array}{l}\text { Country/ } \\
\text { Countries }\end{array}$ & $\begin{array}{l}\text { Point } \\
\text { of view }\end{array}$ & Accessibility & Methodology & $\begin{array}{l}\text { Definition e-learning } \\
\text { platforms, } \\
\text { instruments and/or } \\
\text { devices used }\end{array}$ & $\begin{array}{l}\text { Total no. of } \\
\text { subjects } \\
\text { (Total no. of } \\
\text { subjects with } \\
\text { LD) }\end{array}$ & $\begin{array}{l}\text { Type of } \\
\text { school/ } \\
\text { university }\end{array}$ & Kind of LD & Age range & $\begin{array}{l}\text { Assessed } \\
\text { Variables }\end{array}$ \\
\hline $\begin{array}{l}\mathbf{1 6 .} \\
\text { Richardson, } \\
2016\end{array}$ & $\begin{array}{l}\text { This study examined } \\
\text { the experiences of } \\
\text { students taking the } \\
\text { same courses in the } \\
\text { humanities by distance } \\
\text { learning when tutorial } \\
\text { support was provided } \\
\text { conventionally (using } \\
\text { limited face-to-face } \\
\text { sessions with some } \\
\text { contact by telephone } \\
\text { and email) or online } \\
\text { (using a combination of } \\
\text { computer-mediated } \\
\text { conferencing and } \\
\text { email). }\end{array}$ & UK & S & yes & & $\begin{array}{l}\text { In distance learning, the } \\
\text { curriculum was } \\
\text { traditionally provided } \\
\text { through } \\
\text { correspondence } \\
\text { materials. Nevertheless, } \\
\text { most distance-learning } \\
\text { institutions use various } \\
\text { kinds of personal } \\
\text { support in trying to } \\
\text { narrow what Moore } \\
\text { (1980) called the } \\
\text { "transactional distance" } \\
\text { with their students, } \\
\text { most commonly } \\
\text { through regular albeit } \\
\text { limited tutorials. In } \\
\text { recent years, there has } \\
\text { been an increasing use } \\
\text { of information } \\
\text { technology in distance } \\
\text { education, with a move } \\
\text { from paper-based to } \\
\text { electronic materials } \\
\text { accompanied by a } \\
\text { move from face-to-face } \\
\text { to online tutorial } \\
\text { support }\end{array}$ & $292(24)$ & $U$ & Dyslexia & n.s. & $\begin{array}{l}\text { The results showed } \\
\text { that, given a choice } \\
\text { between } \\
\text { face-to-face and } \\
\text { online tutorial } \\
\text { support, students } \\
\text { with and without } \\
\text { disabilities were } \\
\text { equally likely to } \\
\text { choose online } \\
\text { support rather than } \\
\text { face-to-face } \\
\text { support }\end{array}$ \\
\hline $\begin{array}{l}\text { 17. Sharabi } \\
\text { et al., } 2016\end{array}$ & $\begin{array}{l}\text { This study investigates } \\
\text { personal resources, } \\
\text { loneliness, and } \\
\text { academic self-efficacy } \\
\text { among college } \\
\text { students with and } \\
\text { without LD in } \\
\text { smartphone and } \\
\text { internet use }\end{array}$ & Israel & S & No & Questionnaire & Smart phones Internet & $178(59)$ & $\begin{array}{l}\text { Transition } \\
\text { to college }\end{array}$ & n.s. & 24 mean age & $\begin{array}{l}\text { Coping, } \\
\text { Self-efficacy } \\
\text { Predictors of } \\
\text { loneliness Hope, } \\
\text { optimism Sense of } \\
\text { coherence }\end{array}$ \\
\hline
\end{tabular}


TABLE 1 | Continued

\begin{tabular}{|c|c|c|c|c|c|c|c|c|c|c|c|}
\hline $\begin{array}{l}\text { Author(s), } \\
\text { Year }\end{array}$ & $\begin{array}{l}\text { Topic of } \\
\text { paper }\end{array}$ & $\begin{array}{l}\text { Country/ } \\
\text { Countries }\end{array}$ & $\begin{array}{l}\text { Point } \\
\text { of view }\end{array}$ & Accessibility & Methodology & $\begin{array}{l}\text { Definition e-learning } \\
\text { platforms, } \\
\text { instruments and/or } \\
\text { devices used }\end{array}$ & $\begin{array}{l}\text { Total no. of } \\
\text { subjects } \\
\text { (Total no. of } \\
\text { subjects with } \\
\text { LD) }\end{array}$ & $\begin{array}{l}\text { Type of } \\
\text { school/ } \\
\text { university }\end{array}$ & Kind of LD & Age range & $\begin{array}{l}\text { Assessed } \\
\text { Variables }\end{array}$ \\
\hline $\begin{array}{l}18 . \\
\text { Shonfeld } \\
\text { and Ronen, } \\
2015\end{array}$ & $\begin{array}{l}\text { This study investigates } \\
\text { how adapting the } \\
\text { online course by using } \\
\text { information and } \\
\text { communication } \\
\text { technology following } \\
\text { formative assessment } \\
\text { will improve students' } \\
\text { self-learning ability as } \\
\text { well as broaden their } \\
\text { science knowledge, } \\
\text { their lab performance } \\
\text { and teaching skills. The } \\
\text { study was focused on } \\
\text { preparing K-2 } \\
\text { pre-service teachers }\end{array}$ & n.s. & S & No & $\begin{array}{l}\text { Questionnaires and } \\
\text { interviews }\end{array}$ & $\begin{array}{l}\text { Online learning as a } \\
\text { teaching tool, the } \\
\text { challenge of adapting a } \\
\text { course for three groups } \\
\text { of students: students } \\
\text { with learning } \\
\text { disabilities, excellent } \\
\text { students, and average } \\
\text { students }\end{array}$ & $121(25)$ & $u$ & n.s. & n.s. & $\begin{array}{l}\text { The online course } \\
\text { was based on the } \\
\text { Highlearn platform } \\
\text { which enabled ICT } \\
\text { learning } \\
\text { synchronously } \\
\text { through InterWise. } \\
\text { The course } \\
\text { included peer } \\
\text { teaching: students } \\
\text { conducted group } \\
\text { discussion and } \\
\text { peer feedback; } \\
\text { individual } \\
\text { monitored learning. } \\
\text { All students were } \\
\text { instructed by the } \\
\text { lecturer in } \\
\text { developing }\end{array}$ \\
\hline $\begin{array}{l}\text { 19. Smith } \\
\text { et al., } 2016\end{array}$ & $\begin{array}{l}\text { This study investigated } \\
\text { parent perceptions and } \\
\text { experiences regarding } \\
\text { fully online learning for } \\
\text { their children with } \\
\text { disabilities }\end{array}$ & Kansas & $\mathrm{P}$ & Yes & Interviews & $\begin{array}{l}\text { Online learning in online } \\
\text { schools }\end{array}$ & $\begin{array}{l}18 \text { (Parents of } \\
\text { children) (7) }\end{array}$ & S & $\begin{array}{l}\text { Learning } \\
\text { disabilities }\end{array}$ & n.s. & $\begin{array}{l}\text { Parental role on } \\
\text { online learning } \\
\text { Communication }\end{array}$ \\
\hline $\begin{array}{l}\text { 20. Straub } \\
\text { and } \\
\text { Vasquez, } \\
2015\end{array}$ & $\begin{array}{l}\text { This study investigates } \\
\text { online writing } \\
\text { instruction for students } \\
\text { with learning disabilities } \\
\text { (LDs) using } \\
\text { synchronous online } \\
\text { collaborative writing } \\
\text { software to investigate } \\
\text { effects of self-regulated } \\
\text { strategy development } \\
\text { for strategy instruction } \\
\text { in persuasive writing. }\end{array}$ & USA Florida & S & No & n.s. & $\begin{array}{l}\text { Writing Instruction } \\
\text { Online }\end{array}$ & $4(4)$ & $S$ & n.s. & adolescent & $\begin{array}{l}\text { Self-regulated } \\
\text { strategies } \\
\text { development }\end{array}$ \\
\hline
\end{tabular}




\begin{tabular}{|c|c|c|c|c|c|c|c|c|c|c|c|}
\hline $\begin{array}{l}\text { Author(s), } \\
\text { Year }\end{array}$ & $\begin{array}{l}\text { Topic of } \\
\text { paper }\end{array}$ & $\begin{array}{l}\text { Country/ } \\
\text { Countries }\end{array}$ & $\begin{array}{l}\text { Point } \\
\text { of view }\end{array}$ & Accessibility & Methodology & $\begin{array}{l}\text { Definition e-learning } \\
\text { platforms, } \\
\text { instruments and/or } \\
\text { devices used }\end{array}$ & $\begin{array}{l}\text { Total no. of } \\
\text { subjects } \\
\text { (Total no. of } \\
\text { subjects with } \\
\text { LD) }\end{array}$ & $\begin{array}{l}\text { Type of } \\
\text { school/ } \\
\text { university }\end{array}$ & Kind of LD & Age range & $\begin{array}{l}\text { Assessed } \\
\text { Variables }\end{array}$ \\
\hline $\begin{array}{l}\text { 21. Terras } \\
\text { et al., } 2015\end{array}$ & $\begin{array}{l}\text { This study investigated } \\
\text { how online learning } \\
\text { may afford students } \\
\text { with disabilities } \\
\text { enhanced opportunities } \\
\text { for academic success. } \\
\text { In this study, the } \\
\text { authors interviewed } 11 \\
\text { graduate students to } \\
\text { determine their } \\
\text { experiences with } \\
\text { disability } \\
\text { accommodations in } \\
\text { online courses and their } \\
\text { perceptions of the } \\
\text { relationship between } \\
\text { those accommodations } \\
\text { and their academic } \\
\text { success. }\end{array}$ & $\begin{array}{l}\text { North } \\
\text { Dakota }\end{array}$ & S & Yes & n.s. & $\begin{array}{l}\text { Accommodation in } \\
\text { online courses Since } \\
\text { students with } \\
\text { disabilities may have } \\
\text { difficulty concentrating, } \\
\text { staying on task, and } \\
\text { adhering to a schedule, } \\
\text { online settings } \\
\text { (particularly those that } \\
\text { are asynchronous) } \\
\text { allow students to } \\
\text { access courses } \\
\text { anywhere, anytime, and } \\
\text { any place and provide } \\
\text { "the personalized time } \\
\text { they need to think, } \\
\text { process, and respond". }\end{array}$ & $\begin{array}{l}11 \text { (4 LD } 2 \\
\text { ADHD) }\end{array}$ & $U$ & $\begin{array}{l}\text { Learning } \\
\text { disabilities } \\
\text { ADHD }\end{array}$ & $22-55$ & $\begin{array}{l}\text { Disability } \\
\text { accommodations in } \\
\text { online courses } \\
\text { students } \\
\text { responsibility } \\
\text { instructor } \\
\text { responsibility } \\
\text { University } \\
\text { responsibility }\end{array}$ \\
\hline $\begin{array}{l}\text { 22. Vasalou } \\
\text { et al., } 2017\end{array}$ & $\begin{array}{l}\text { This study investigates } \\
\text { the case of a digital } \\
\text { game called Words } \\
\text { Matter. The game was } \\
\text { designed for children } \\
\text { with dyslexia and was } \\
\text { informed by principles } \\
\text { from casual games and } \\
\text { evidence-based } \\
\text { practice from special } \\
\text { education. Focusing on } \\
\text { the game play of two } \\
\text { groups of children, we } \\
\text { employ a systematic } \\
\text { thematic analytic } \\
\text { approach on videos of } \\
\text { children's verbal and } \\
\text { non-verbal interaction } \\
\text { triangulated with their } \\
\text { game logs, } \\
\text { concentrating on the } \\
\text { nature of } \\
\text { student-student as well } \\
\text { as student-tutor social } \\
\text { interactions. }\end{array}$ & UK & $S$ & No & Case studies & $\begin{array}{l}\text { Drill and practice digital } \\
\text { games-based learning } \\
\text { Games-based } \\
\text { pedagogies for } \\
\text { students with special } \\
\text { education needs }\end{array}$ & $8(8)$ & S & Dyslexia & $11-12$ & $\begin{array}{l}\text { Motivation } \\
\text { Engagement on } \\
\text { learning Social } \\
\text { engagement } \\
\text { Self-esteem } \\
\text { Personal identity } \\
\text { Peer tutoring }\end{array}$ \\
\hline
\end{tabular}




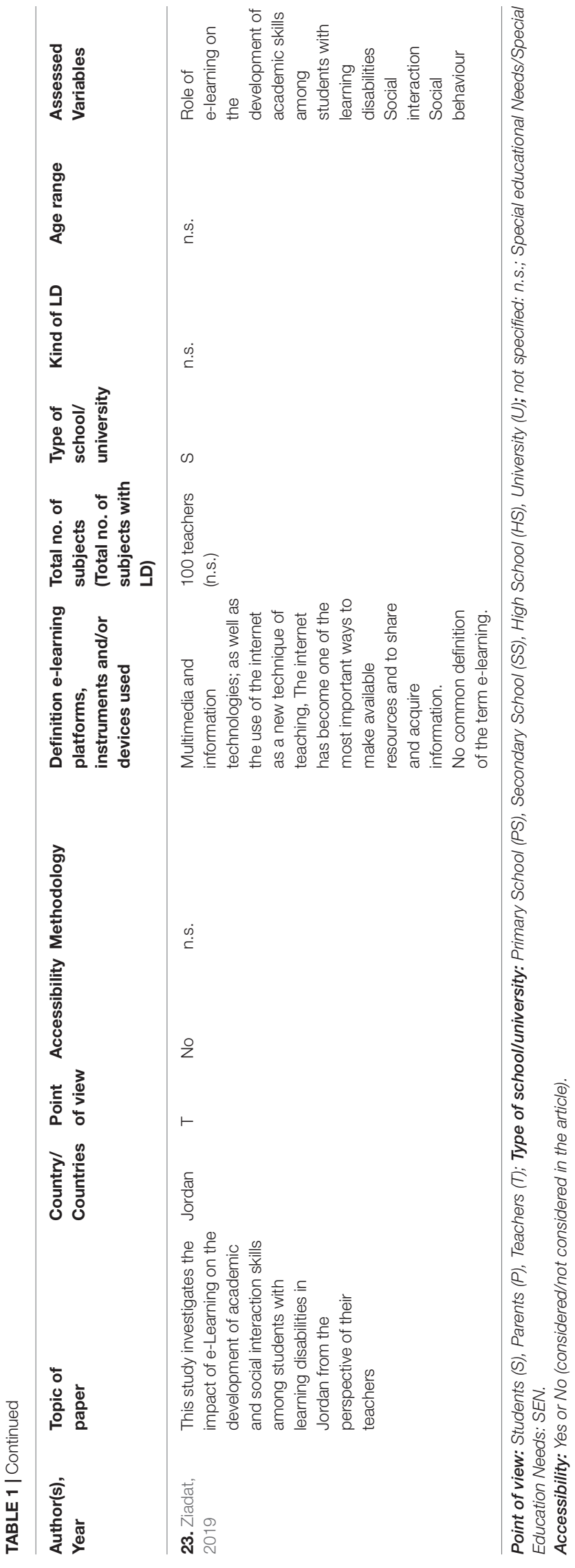

other 1/2 examined primary and secondary schools (Chen et al., 2015; Straub and Vasquez, 2015; Benmarrakchi et al., 2017; Rice and Carter, 2016; Smith et al., 2016; Adam and Tatnall, 2017; Berizzi et al., 2017; Vasalou et al., 2017; Baharuddin and Dalle, 2019; Lipka et al., 2019; Ouherrou et al., 2019; Ziadat, 2019; Nieto-Márquez et al., 2020). One paper focused on the transition from school to university (Sharabi et al., 2016). As expected, we also found a considerable heterogeneity in school settings, ranging from mainstream school/classrooms to special needs schools/classrooms, according to specific national and theoretical approaches and policies regarding the field of inclusion (see Table 2). Given that the countries in our sample ranged across Europe, United States, as well as Arab and Slavic countries, there was some diversity in the idea of inclusive policies for students with LDs. This is due to national differences regarding the issues of policies for students with LDs and, in general, for students with SEN. In some countries, there is an inclusion-based approach where students with LDs are placed in mainstream schools; in other countries there are special schools and special classrooms for them. In some countries, transition to complete inclusion is still ongoing (Lindsay, 2016; Norwich, 2016; Petretto et al., 2019; Pilia, 2019). While one of the papers described a specific experience in two special needs classes (Adam and Tatnall, 2017), other research papers concentrated on the use of specific e-learning approaches to designated groups of children with LDs or to all the children in the classroom in mainstream schools (Straub and Vasquez, 2015; Vasalou et al., 2017).

The approaches employed range from the use of specific devices and/or platforms, to the use of specific "reasonable accommodations" (such as font quality and sizes in the learning materials on the web or the use of specific support technologies) (Chen et al., 2015; Benmarrakchi et al., 2017; Rice and Carter, 2016; Alamri and Tyler-Wood, 2017; Berizzi et al., 2017; Ouherrou et al., 2019; García-González et al., 2020); or the use of software/games aimed to increase specific abilities in students with LDs (Straub and Vasquez, 2015; Vasalou et al., 2017). For university settings, some articles describe the experiences of socalled "Open universities" that have been based on distance learning methods since they started. With the development of ICTs, in the past few decades these universities have started to use e-learning platforms to contact students and to promote learning and social connections (Richardson, 2015, 2016; Kent et al., 2018). Their ongoing experiences focus mainly on the attainment of students with LDs as well as on the need to increase access to information and learning. Other studies focus on the need for dedicated online courses to specific categories of students, aiming at reducing barriers and distances and providing specific accommodations (Terras et al., 2015).

The age range in these university samples is very wide. From a positive perspective it can represent a sign of the wider opportunity for older people to access university courses. However, according to some studies, it could be also the sign of a lower and slower attainment of students with LDs in University (Richardson, 2015, 2016; Shonfeld and Ronen, 2015). The topics of attainment and achievement are interesting because even though some papers have discussed the risk of low achievement for students with LDs, other studies 
have demonstrated the positive effect of accommodations and have showed examples of unexpected achievement by LD students (Shonfeld and Ronen, 2015). Another aspect is the fear of disclosure of their diagnosis by some students with LDs and the effects on their tendency to hide diagnoses rather than to communicate it, even when they should do so in order to define specific "reasonable accommodations" (Richardson, 2015, 2016; Terras et al., 2015). Although there may be increased student awareness of the need to disclose their diagnosis and the functional profiles that help to define a personalized approach that facilitates their access to learning and materials, some authors have highlighted the importance of further discussing the role of communication between teachers/instructors and students with LDs in the development of more comfortable learning environments and in the pursuit of shared learning and achievement aims (Terras et al., 2015).

\section{Focus on Psychological Well-Being}

Few studies have directly examined the psychological aspects of students with LDs in e-environments. Some papers have focused on psychological consequences of the intensified use of Information and Communications Technologies (ICTs); other papers instead focused especially on adults, addressing some psychological effects of e-learning procedures adapted to students with LDs. In their study, Ouherrou et al. (2019) highlighted the fact that the integration of ICTs in special needs education may have a positive impact on the emotional states of children with LDs, because they may experience fewer negative emotions than findings of current literature would suggest with regard to the presence of higher levels of negative emotions in the classroom. Vasalou et al. (2017) argued that a socially constructed view of digital games-based learning provides new opportunities for the support of children with dyslexia. Children spontaneously engage in "game talk" regarding game performance, content, actions and they strategically use their individual game experiences to express their personality and interact with their peers. Also, such experiences can help improve the intra-individual function by enhancing a child's self-esteem. The findings of Sharabi et al. (2016) supported earlier studies that assessed children and adolescents with LDs (Sharabi and Margalit, 2014), showing that college students with LDs possess lower levels of personal resources (sense of coherence, hope and academic self-efficacy) and suffer higher levels of social distress and loneliness than their peers. The loneliness factor was predicted by measuring online avoidance coping, their amount of smartphone use and by examining their personal resources, the use of ICTs may provide additional environmental conditions to enable youngsters to meet their emotional needs. At the same time, these opportunities may also be misused as avoidance coping and thus may contribute to increased loneliness and lower academic self-efficacy. Coherently with previous studies, Lambert and Dryer (2018) highlighted that

TABLE 2 | Papers which met the inclusion criteria in the school setting analyzed according to Bjekic et al. (2014).

\begin{tabular}{|c|c|c|c|c|}
\hline \multirow[t]{2}{*}{ School } & \multicolumn{2}{|c|}{ E-learning (N Studies) } & \multirow[t]{2}{*}{ Limitations } & \multirow[t]{2}{*}{ Strenghts } \\
\hline & ICT & E-platforms & & \\
\hline $\begin{array}{l}\text { Primary/Secondary } \\
\text { School }\end{array}$ & $4\left(3^{\star}\right)$ & $8^{\star \star}\left(6^{\star}\right)$ & $\begin{array}{l}\text { - Lack of interest: } \\
\text { - technology in the development of } \\
\text { student curriculum } \\
\text { design framework for digital materials } \\
\text { personalized paths } \\
\text { parent's training in supporting children's } \\
\text { e-learning experience }\end{array}$ & $\begin{array}{l}\text { - Promotion of: } \\
\text { - skills and academic knowledge } \\
\text { educational outcome } \\
\text { students'independence and self-regulation } \\
\text { pathway for the transition from school to further } \\
\text { study } \\
\text { learning styles } \\
\text { communication among children, teachers and } \\
\text { parents } \\
\text { metacognitive experience } \\
\text { emotional well-being } \\
\text { tutor/teacher scaffolding } \\
\text { parents support }\end{array}$ \\
\hline High school & $1^{\star}$ & $1^{\star}$ & $\begin{array}{l}\text { - Lack of: } \\
\text { - design interface } \\
\text { appropriate online instruction strategies }\end{array}$ & $\begin{array}{l}\text { - Promotion of: } \\
\text { - tutor/teacher scaffolding } \\
\text { social support } \\
\text { stress and anxiety reduction }\end{array}$ \\
\hline University & & $12\left(3^{\star}\right)$ & $\begin{array}{l}\text { - Lack of: } \\
\text { - longitudinal studies } \\
\text { self-regulation and emotional well-being }\end{array}$ & $\begin{array}{l}\text { - Promotion of: } \\
\text { - scaffolding (instructor-learner interaction) } \\
\text { social support } \\
\text { metacognitive interventions } \\
\text { academic retention }\end{array}$ \\
\hline
\end{tabular}

* Presence of Assistive Technology.

** Three studies with E-platforms and ICT. 
in high education the e-environment had a negative influence on the quality of life of students with increased stress and anxiety, the perception of feelings of inadequacy, a decrease in time available for other activities and personal relationships. The same authors also highlighted that for many students, the academic and emotional support provided by family and friends was a key factor in study success. Studies on the perception of the impact of e-learning on the development of academic skills and social interaction from the perspective of students and/or teachers showed that the quality of teacher-student relationships contribute to producing improvements in learning achievement (Alamri and Tyler-Wood, 2017; Lipka et al., 2019; Ziadat, 2019). Only a small number of studies have considered the role of parents. Smith et al. (2016) investigated parents' perceptions and experiences regarding exclusive online learning for their children with disabilities. The results showed that this experience altered parents' previous roles and that many parents were not equipped to take a teaching role due to lack of training, time, and other constraints. A parent-as-teacher role can negatively affect parent-child dynamics, leading to frustration for parent and child but full online learning requires increased parent-teacher communication. This increased level of interaction and the positive outcomes associated with the shared information enhanced a collaborative parent-teacher relationship. The use of ICT and e-learning can improve the learning of students with LDs only where a supportive context is present. The support provided by family, teachers and peers can create a protective factor which improves the well-being of students with LDs.

\section{Focus on the Accessibility Standards and Emotional Distress}

Many of the difficulties in designing e-learning courses are due to accessibility issues that can affect successful engagement (Draffan, 2012; Seale, 2013). The heterogeneity of the LD population entails great challenges to all parties involved in creating, managing and using e-learning content, tools and platforms with accessibility features (Guenaga et al., 2004; Baharuddin and Dalle, 2019). Some papers described the risks of a design approach based on a general and average idea of students without LDs (Kent et al., 2018). For Beacham and Alty (2006) the e-learning materials commonly employed were developed with the needs and capabilities of non-dyslexic learners in mind; clearly, resources do not generally take into consideration the individual learning approaches that these students manifest (Alsobhi and Abeysinghe, 2013; Chen et al., 2015; Luongo, 2018). Chen et al. (2015) also underline this point, observing that empirically derived guidelines for designing accessible online learning environments for learners with dyslexia are still scarce. The problem of accessibility is fundamental in e-learning design, as it is strictly linked to certain psychological factors that will affect students, like willingness to focus on learning, management of emotions and behavior, learning motivation, interest and self-regulation (Chen et al., 2015; Berizzi et al., 2017; Luongo, 2018).
Existing literature provides clear evidence that text-based synchronous activities commonly used in education, like chat programs and videoconference, can create psychological and learning difficulties. However, only a small number of papers take into account the problems of students with LDs in collaborative environments (Luongo, 2018). Some papers focus on the positive aspects of the use of e-learning platforms in increasing accessibility to information and learning materials (Richardson, 2016), above all because participation in remote activities, like on-line forum discussions, improves the autonomy and self-regulation of students (Berizzi et al., 2017). These aspects are reinforced by continuous support of tutors and peers, and reflection on what has been done, the goals to be achieved, and ultimately the strategies to be adopted. Other articles described the possible role of a "universal design for learning approach" in the design of websites, web materials and e-learning platforms (Chen et al., 2015; Shonfeld and Ronen, 2015; Alamri and TylerWood, 2017; Kent et al., 2018; Nieto-Márquez et al., 2020) in order to create environments that can be useful also for students with LDs.

\section{CONCLUSION}

This mini-review has attempted to analyze both the quality of life of students with LDs and their interpersonal relationships and the features of e-learning that can have positive and negative effects on them. The considerable heterogeneity of the articles we selected led us to the following reflections: we are aware that the heterogeneity could represent a limit but also an expected consequence of the chosen way of to explore a complex topic. Bearing in mind this issue, in a following article we will discuss the picture of the state of art that we derived from this minireview. In the near future, we will explore specific and more focused aspects, also with an attention on intervention aims. Two issues are emerged.

The first is how important online-support is to consolidate teacher-learner relationships, as it can affect a student's wellbeing and learning achievement. We know that e-learning is a psychological process supported by e-technology, and learning is a social activity. Understanding that it is socially constructed should ensure that e-learning is organized to promote participation, allowing all students to take part in all activities, thus enhancing cooperative-learning.

The second consideration regards the fundamental role of accessibility and "reasonable accommodations", which should lead to a reduction of emotional distress and promote positive psychological factors through full engagement with e-learning. In order to be effective, e-learning must go beyond simply digitizing books and ought to be designed carefully and appropriately for learners (Penna and Stara, 2007, 2010). What about the current and ongoing experience of the massive use of e-learning due to the COVID-19 outbreak? We agree with Al Lily et al. (2020), 
who coined the term "Crisis Distance learning," that the current ongoing experience is different from previous ones, and that caution is needed before making any kind of generalizations from previous experiences. Nevertheless, some general considerations can be drawn for future research. It is necessary to encourage and maintain cooperative approaches in all spheres, including in the use of e-learning in school and universities, with particular attention on the quality of the relationships between all the people involved (students-teachers-parents-peers) and with an even more specific focus on the psychological needs of students with LDs. The improvement of e-learning systems designed with attention to the care and quality of relationships can promote well-being among all parties involved in the learning process.

\section{REFERENCES}

Adam, T., and Tatnall, A. (2017). The value of using ICT in the education of school students with learning difficulties. Educ. Inf. Technol. 22, 2711-2726. doi: $10.1007 /$ s10639-017-9605-2

Al Lily, A. E., Ismail, A. F., Abunasser, F. M., and Alqahtani, R. H. A. (2020). Distance education as a response to pandemics: coronavirus and Arab culture. Technol. Soc. 63:101317. doi: 10.1016/j.techsoc.2020.101317

Alamri, A., and Tyler-Wood, T. (2017). Factors affecting learners with disabilitiesinstructor interaction in online learning. JSET 32, 59-69. doi: 10.1177/ 0162643416681497

Alsobhi, A. Y., and Abeysinghe, G. (2013). "An evaluation of accessibility of e-learning for dyslexic students," in Proceedings of the 2013 International Conference on Current Trends in Information Technology (CTIT), (Piscataway, NJ: IEEE), 1-4. doi: 10.1109/CTIT.2013.6749468

American Psychiatric Association (2013). Diagnostic and Statistical Manual of Mental Disorders DSM5. Washington DC: APA. doi: 10.1176/appi.books. 9780890425596

Baharuddin, B., and Dalle, J. (2019). Transforming learning spaces for elementary school children with special needs. JSSER 10, 344-365.

Beacham, N. A., and Alty, J. L. (2006). An investigation into the effects that digital media can have on the learning outcomes of individuals who have dyslexia. Comput. Educ. 47, 74-93. doi: 10.1016/j.compedu.2004.10.006

Benmarrakchi, F., El Kafi, J., Elhore, A., and Haie, S. (2017). Exploring the use of the ICT in supporting dyslexic students' preferred learning styles: a preliminary evaluation. Educ. Inf. Technol. 22, 2939-2957. doi: 10.1007/s10639-016-9551-4

Berizzi, G., Di Barbora, E., and Vulcani, M. (2017). Metacognition in the e-learning environment: a successful proposition for Inclusive Education. Je-LKS 13, 47-57. doi: 10.20368/1971-8829/1381

Bjekic, D., Obradovic, S., Vucetic, M., and Bojovic, M. (2014). E-teacher in inclusive e-education for students with specific learnind disabilities. Procedia Soc. Behav. Sci. 128, 128-133. doi: 10.1016/j.sbspro.2014.03.131

Bonifacci, B., Storti, M., Tobia, V., and Suardi, A. (2016). Specific learning disorders: a look inside children's and parents' psychological well-being and relationships. J. Learn. Disabil. 49, 532-545. doi: 10.1177/0022219414566681

Chen, C., Keong, M., Teh, C., and Chuah, K. (2015). Learners with Dyslexia: exploring their experiences with different online reading affordances. Themes Sci. Technol. Educ. 8, 63-79.

Cidral, W. A., Oliveira, T., Di Felice, M., and Aparicio, M. (2018). E-learning success determinants: Brazilian empirical study. Comput. Educ. 122, 273-290. doi: 10.1016/j.compedu.2017.12.001

Draffan, E. A. (2012). "Dyslexia, elearning and eskills," in Supporting Dyslexic Adults in Higher Education and the Workplace, ed. N. Brunswick (Hoboken, NJ: John Wiley \& Sons, Ltd), 84-90. doi: 10.1002/9781119945000.ch9

Eletti, V. (2002). Che cos'è l'e-Learning. Roma: Carocci editore.

Filippello, P., Buzzai, C., Messina, G., Mafodda, A. V., and Sorrenti, L. (2019). School refusal in students with low academic performances and Specific Learning Disorder. the role of self-esteem and perceived parental psychological control. Intl. J. Disabil. Dev. Educ. 67, 592-607. doi: 10.1080/1034912x.2019. 1626006

\section{AUTHOR CONTRIBUTIONS}

All authors equally contributed to the design of the study. All authors have read and agreed to the published version of the manuscript.

\section{FUNDING}

This work was supported by ATS Sardinia: title project "Profilo Neuro-Psicologico e Problematiche Emotive nei DSA: Una Proposta di Ricerca-Intervento" - "Neuro-Psychological Profile and Emotional Problems in LDs: A Research-Intervention Proposal" (November, 2019; June 2021).

Frith, U. (2013). Autism and Dyslexia: a glance over 25 years of research. Perspect. Psychol. Sci. 8, 670-672. doi: 10.1177/1745691613507457

García-González, J. M., Gómez-Calcerrada, S. G., Solera Hernández, E., and RíosAguilar, S. (2020). Barriers in higher education: perceptions and discourse analysis of students with disabilities in Spain. Disab. Soc. doi: 10.1080/09687599. 2020.1749565

Guenaga, M. L., Burger, D., and Oliver, J. (2004). “Accessibility for e-learning environments," in Proceedings of the International Conference on Computers for Handicapped Persons. Berlin: Springer, 157-163. doi: 10.1007/978-3-54027817-7_23

Kent, M., Ellis, K., and Giles, M. (2018). Students with Disabilities and eLearning in Australia: experiences of accessibility and disclosure at Curtin University. TechTrends 62, 654-663. doi: 10.1007/s11528-018-0337-y

Lambert, D. C., and Dryer, R. (2018). Quality of life of higher education students with learning disability studying online. Int. J. Disabil. Dev. Educ. 65, 393-407. doi: 10.1080/1034912X.2017.1410876

Lindsay, G. (2016). Grand Challenge: priorities for research in Special Educational Needs. Front. Educ. 1:1. doi: 10.3389/feduc.2016.00001

Lipka, O., Baruch, F. A., and Meer, Y. (2019). Academic support model for postsecondary school students with learning disabilities: student and instructor perceptions. Int. J. Incl. Educ. 23, 142-157. doi: 10.1080/13603116.2018. 1427151

Luongo, N. (2018). An examination of distance learning faculty satisfaction levels and self-perceived Barriers. J. Educ. Online 15:12. doi: 10.9743/jeo.2018.15.2.8

Moher, D., Shamseer, L., and Clarke, M. (2015). Preferred reporting items for systematic review and meta-analysis protocols (PRISMA-P) 2015 statement. Syst. Rev. 4:1. doi: 10.1186/2046-4053-4-1

Naumova, T. A., Vytovtova, N. I., Miyiukov, N. W., and Zulfugarzade, T. E. (2017). Model of distant learning educational methods for the students with disabilities. Eur. J. Contemp. Educ. 6, 565-573. doi: 10.13187/ejced.2017. 3.565

Nieto-Márquez, L. N., Baldominos, A., and Pérez-Nieto, M. Á (2020). Digital teaching materials and their relationship with the metacognitive skills of students in primary education. Educ. Sci. 10:113. doi: 10.3390/educsci100 40113

Norwich, B. (2016). Conceptualizing special educational needs using a biopsychosocial model in England: the prospects and challenges of Using the International Classification of Functioning Framework. Front. Educ. 1:5. doi: 10.3389/feduc.2016.00005

Ouherrou, N., Elhammoumi, O., Benmarrakchi, F., and El Kafi, J. (2019). Comparative study on emotions analysis from facial expressions in children with and without learning disabilities in virtual learning environment. Educ. Info. Technol. 24, 1777-1792. doi: 10.1007/s10639-018-09852-5

Panicker, A. S., and Chelliah, A. (2016). Resilience and stress in children and adolescents with specific learning disability. JCACAP 25, 17-23.

Penna, M. P., and Stara, V. (2007). The failure of e-learning: why should we use a learner centred design. Je-LKS 3, 127-135.

Penna, M. P., and Stara, V. (2010). Opinions on computers, and efficacy of a computer-based learning: a pilot study. Educ. Info. Technol. 15, 181-204. doi: 10.1007/s10639-009-9104-1 
Petretto, D. R., and Masala, C. (2017). Dyslexia and specific learning disorders: new international diagnostic Criteria. J. Child Dev. Disord. 3:19. doi: 10.4172/24721786.100056

Petretto, D. R., Pilia, R., Volterra, S., and Masala, C. (2019). "Bisogni Educativi Speciali: uno sguardo sulla complessità," in I Bisogni Educativi Speciali: il diritto Allistruzione in una Prospettiva Inclusiva, eds D. R. Petretto, F. Bariffi, E. Jimenez, S. Volterra, R. Pilia, and C. Masala (Via Mezzocannone: Edizioni Jovene).

Pilia, R. (2019). "Special educational needs and additional support for learning: il modello dell'inclusione scolastica in Scozia," in I Bisogni Educativi Speciali: il diritto Allistruzione in Una Prospettiva Inclusiva, eds D. R. Petretto, F. Bariffi, E. Jimenez, S. Volterra, R. Pilia, and C. Masal (Via Mezzocannone: Edizioni Jovene).

Rice, M. F., and Carter, R. A. (2016). Online teachers work to support self regulation of learning in students with disabilities at a fully online state virtual school. Online Learn. 20, 118-135. doi: 10.24059/olj.v20i4.1054

Richardson, J. T. E. (2015). Academic attainment in students with Dyslexia in distance education. Dyslexia 21, 323-337. doi: 10.1002/dys.1502

Richardson, J. T. E. (2016). Face-to-Face versus online tutorial support in distance education: preference, performance, and pass rates in students with disabilities. JPED 29, 83-90.

Salehi, H., Shojaee, M., and Sattar, S. (2015). Using E-Learning and ICT courses in educational environment: a review. Engl. Lang. Teach. 8, 63-70. doi: /10.5539/ elt.v8n1p63

Schulte-Korne, G. (2014). Specific learning disabilities from DSM-IV to DSM-5. Z. Kinder Jugendpsychiatr. Psychother. 42, 369-372; quiz 373-4. doi: 10.1024/ $1422-4917 / \mathrm{a} 000312$

Seale, J. K. (2013). E-learning and Disability in Higher Education: Accessibility Research and Practice. Abingdon: Routledge. doi: 10.4324/9780203095942

Sharabi, A., and Margalit, M. (2014). Predictors of positive mood and negative mood among children with learning disabilities (LD) and Their Peers. IJRLD $2,18-41$.

Sharabi, A., Sade, S., and Margalit, M. (2016). Virtual connections, personal resources, loneliness, and academic self-efficacy among college students with and without LD. Eur. J. Spec. Needs Educ. 31, 376-390. doi: 10.1080/08856257. 2016.1141542

Shonfeld, M., and Ronen, I. (2015). Online learning for students from diverse backgrounds: learning disability students, excellent students and average students. IAFOR J. Educ. 3, 13-29. doi: 10.22492/ije.3.2.01

Smith, S. J., Burdette, P. J., Cheatham, G. A., and Harvey, S. P. (2016). Parental role and support for online learning of students with disabilities: a paradigm shift. JSEL 29, 101-112.
Sorrenti, L., Spadaro, L., Mafodda, A. V., Scopelliti, G., Orecchio, S., and Filippello, P. (2019). The predicting role of school Learned helplessness in internalizing and externalizing problems. an exploratory study in students with Specific Learning Disorder. Mediterr. J. Clin. Psychol. 7, 1-14.

Straub, C., and Vasquez, E. (2015). Effects of synchronous online writing instruction for students with learning disabilities. JSET 30, 213-222. doi: 10. $1177 / 0162643415618929$

Terras, K., Leggio, J., and Phillips, A. (2015). Disability accommodations in online courses: the graduate student experience. JPED 28, 329-340.

Vasalou, A., Khaled, R., Holmes, W., and Gooch, D. (2017). Digital gamesbased learning for children with dyslexia: a social constructivist perspective on engagement and learning during group game-play. Comput. Educ. 114, 175-192. doi: 10.1016/j.compedu.2017.06.009

Viner, R. M., Russell, S. J., Croker, H., Packer, J., Ward, J., Stansfield, C., et al. (2020). School closure and management practices during coronavirus outbreaks including COVID-19: a rapid systematic review. Lancet. Child Adolesc. 4, 397-404. doi: 10.1016/S2352-4642(20)30095-X

Visser, L., Kalmar, J., Linkersdorfer, J., Gorgen, R., Rother, J., Marcus, H., et al. (2020). Comorbidities between specific learning disorders and psychopathology in elementary school children in Germany. Front. Psychiatry 11:292. doi: 10. 3389/fpsyt.2020.00292

Walker, A., and Nabuzoka, D. (2007). Academic achievement and social functioning of children with without learning difficulties. Educ. Psychol. 27, 635-654. doi: 10.1080/01443410701309175

World Health Organization (2001). International Classification of Functioning, Disability and Health. Geneva: World Health Organization.

Ziadat, A. H. (2019). The impact of e- learning in developing academic skills and social interaction among students with learning disabilities in jordan from the perspective of their teachers. TEM J. 8, 1440-1448. doi: 10.18421/TEM $84-48$

Conflict of Interest: The authors declare that the research was conducted in the absence of any commercial or financial relationships that could be construed as a potential conflict of interest.

Copyright (c) 2021 Cataudella, Carta, Mascia, Masala, Petretto and Penna. This is an open-access article distributed under the terms of the Creative Commons Attribution License (CC BY). The use, distribution or reproduction in other forums is permitted, provided the original author(s) and the copyright owner(s) are credited and that the original publication in this journal is cited, in accordance with accepted academic practice. No use, distribution or reproduction is permitted which does not comply with these terms. 